\title{
ON SYNDETIC RIESZ SEQUENCES
}

\author{
MARCIN BOWNIK AND ITAY LONDNER
}

\begin{abstract}
Applying the solution to the Kadison-Singer problem, we show that every subset $\mathcal{S}$ of the torus of positive Lebesgue measure admits a Riesz sequence of exponentials $\left\{e^{i \lambda x}\right\}_{\lambda \in \Lambda}$ such that $\Lambda \subset \mathbb{Z}$ is a set with gaps between consecutive elements bounded by $\frac{C}{|\mathcal{S}|}$. In the case when $\mathcal{S}$ is an open set we demonstrate, using quasicrystals, how such $\Lambda$ can be deterministically constructed.
\end{abstract}

\section{IntroduCtion}

Let $\mathcal{H}$ be a separable Hilbert space and $I$ a countable set.

Definition 1.1. A system of vectors $\left\{\varphi_{i}\right\}_{i \in I} \subset \mathcal{H}$ is called a frame for $\mathcal{H}$ if there exist positive constants $A \leq B$ such that

$$
A\|f\|_{\mathcal{H}}^{2} \leq \sum_{i \in I}\left|\left\langle f, \varphi_{i}\right\rangle\right|^{2} \leq B\|f\|_{\mathcal{H}}^{2}
$$

for all vectors $f \in \mathcal{H}$.

We say that $\left\{\varphi_{i}\right\}_{i \in I}$ is a Bessel sequence in $\mathcal{H}$ if only the RHS inequality in (1) is satisfied. If $A=B,\left\{\varphi_{i}\right\}_{i \in I}$ is called a tight frame and if $A=B=1$ it is called a Parseval frame.

Definition 1.2. A system of vectors $\left\{\varphi_{i}\right\}_{i \in I} \subset \mathcal{H}$ is called a Riesz sequence in $\mathcal{H}$ if there exist positive constants $A \leq B$ such that

$$
A \sum_{i \in I}\left|a_{i}\right|^{2} \leq\left\|\sum_{i \in I} a_{i} \varphi_{i}\right\|_{\mathcal{H}}^{2} \leq B \sum_{i \in I}\left|a_{i}\right|^{2}
$$

for every finite sequence of scalars $\left\{a_{i}\right\}_{i \in I}$.

In this paper we discuss Riesz sequences of exponentials, i.e. for bounded sets $\mathcal{S}$ of positive Lebesgue measure and a countable set $\Lambda \subset \mathbb{R}$ we consider the exponential system $E(\Lambda)=\left\{e^{i \lambda x}\right\}_{\lambda \in \Lambda}$ in the space $L^{2}(\mathcal{S})$.

Throughout this paper we will denote by $|\mathcal{S}|$ the Lebesgue measure of the set $\mathcal{S}$. Note that if $\Lambda$ is a separated set, i.e. $\inf _{\lambda \neq \mu}|\lambda-\mu|>0$, then the RHS of (1) and (2) is satisfied for $E(\Lambda)$. In particular, if $\mathcal{S} \subset \mathbb{T}=\mathbb{R} / 2 \pi \mathbb{Z}$ is a set of positive Lebesgue measure and $\Lambda \subseteq \mathbb{Z}$,

The first author was supported in part by the NSF grant DMS-1665056. The authors are grateful to Prof. Olevskii for discussions on the subject of this paper. 
then we may take $B=2 \pi$. Also, it follows from Parseval's identity that the normalized exponential system $\frac{1}{\sqrt{2 \pi}} E(\mathbb{Z})$ is a Parseval frame in $L^{2}(\mathcal{S})$.

The case where $I$ is an interval is classical. In order to determine whether a system $E(\Lambda)$ is a Riesz sequence in $L^{2}(I)$, one essentially needs to know the upper density of $\Lambda$

$$
D^{+}(\Lambda)=\lim _{r \rightarrow \infty} \sup _{a \in \mathbb{R}} \frac{\#(\Lambda \cap(a, a+r))}{r} .
$$

Remark. Analogously one may define lower density, and in case these quantities coincide we say $\Lambda$ has uniform density denoted $D(\Lambda)$.

Kahane [7] proved the following result, see also [18].

Theorem $1.3([7])$. If $D^{+}(\Lambda)<\frac{|I|}{2 \pi}$, then $E(\Lambda)$ is a Riesz sequence in $L^{2}(I)$. On the other hand, if $D^{+}(\Lambda)>\frac{|I|}{2 \pi}$, then $E(\Lambda)$ is not a Riesz sequence in $L^{2}(I)$.

For arbitrary sets of positive measure the situation is much more complicated and only necessary condition exists due to Landau [9].

Theorem $1.4([9])$. Let $\mathcal{S} \subset \mathbb{R}$ be a measurable set. If $E(\Lambda)$ is a Riesz sequence in $L^{2}(\mathcal{S})$, then $D^{+}(\Lambda) \leq \frac{|\mathcal{S}|}{2 \pi}$.

In light of Theorem 1.4 it is natural to ask:

Given a set $\mathcal{S}$, does there exist a set $\Lambda$ of positive density such that the exponential system $E(\Lambda)$ is a Riesz sequence in $L^{2}(\mathcal{S})$ ?

This question may be considered under various notions of density. The first result on this subject was obtained by Bourgain and Tzafriri [1, Theorem 2.2] using their celebrated restricted invertibility theorem.

Theorem 1.5 ([1]). Given $\mathcal{S} \subset \mathbb{T}$ of positive measure, there exists a set $\Lambda \subset \mathbb{Z}$ with positive asymptotic density

$$
\operatorname{dens}(\Lambda)=\lim _{r \rightarrow \infty} \frac{\#(\Lambda \cap(-r, r))}{2 r}>c|\mathcal{S}|
$$

and such that $E(\Lambda)$ is a Riesz sequence in $L^{2}(\mathcal{S})$. Here, $c$ is an absolute constant, independent of $\mathcal{S}$.

Theorem 1.5 implies that every set $\mathcal{S}$ admits a Riesz sequence $\Lambda$ with positive upper density, proportional to the measure of $\mathcal{S}$, and so we can answer the question above positively. Another related property is a relatively dense set, also referred to as a syndetic set.

Definition 1.6. A subset $\Lambda=\left\{\ldots<\lambda_{0}<\lambda_{1}<\lambda_{2}<\ldots\right\} \subset \mathbb{Z}$ is called syndetic if the consecutive gaps in $\Lambda$ are bounded, i.e., if there is a positive constant $d$ such that

$$
\lambda_{n+1}-\lambda_{n} \leq \underset{2}{d} \quad \forall n \in \mathbb{Z} .
$$


We denote the smallest possible constant satisfying (3) by $\gamma(\Lambda)$.

The existence of syndetic Riesz sequences has been proved by Lawton [10, Corollary 2.1], and by a different approach by Paulsen [19, Theorem 1.1] to be equivalent to the Feichtinger conjecture.

Theorem $1.7([10])$. Given a set $\mathcal{S} \subset \mathbb{T}$ of positive measure, the following are equivalent:

(i) There exists $r \in \mathbb{N}$ and $\mathbb{Z}=\bigcup_{j=1}^{r} \Lambda_{j}$ such that $E\left(\Lambda_{j}\right)$ is a Riesz sequence in $L^{2}(\mathcal{S})$ for all $j \in[r]=\{1, \ldots, r\}$.

(ii) There exists $d \in \mathbb{N}$ and a syndetic set $\Lambda \subseteq \mathbb{Z}$ with $\gamma(\Lambda)=d$ such that $E(\Lambda)$ is a Riesz sequence in $L^{2}(\mathcal{S})$.

Remark 1.8. While we can trivially deduce $r \leq d$ in the implication (ii) $\Longrightarrow$ (i) by considering translates of $\Lambda$, it should be emphasized that the proof of Theorem 1.7 does not imply any connection in the other direction. Explicitly, knowing the number of partitions $r$ in (i) does not give any upper bound on $\gamma(\Lambda)$ in (ii).

The first statement in Theorem 1.7 is known as the Feichtinger conjecture for exponentials. The Feichtinger conjecture in its general form states that every bounded frame can be decomposed into finitely many Riesz sequences. It has been proved by Casazza et al. in [3] as well as by Casazza and Tremain [4], to be equivalent to the Kadison-Singer problem. The latter has been solved recently by Marcus, Spielman and Srivastava. Their main result is the following theorem [11, Theorem 1.4].

Theorem 1.9 ([11]). If $\varepsilon>0$ and $v_{1}, \ldots, v_{m}$ are independent random vectors in $\mathbb{C}^{d}$ with finite support such that $\sum_{i=1}^{m} \mathbb{E}\left[v_{i} v_{i}^{*}\right]=\mathbf{I}_{d}$ and $\mathbb{E}\left[\left\|v_{i}\right\|^{2}\right] \leq \varepsilon$ for all $i$, then

$$
\mathbb{P}\left(\left\|\sum_{i=1}^{m} v_{i} v_{i}^{*}\right\| \leq(1+\sqrt{\varepsilon})^{2}\right)>0 .
$$

Here and below $\mathbf{I}_{d}$ is the $d \times d$ identity matrix. Later, using similar techniques, Bownik, Casazza, Marcus and Speegle [2, Theorem 1.2] gave an improved bound in the case when random vectors $v_{1}, \ldots, v_{m}$ each have support of size 2 .

Theorem $1.10([2])$. If $\varepsilon \in(0,1 / 2)$ and $v_{1}, \ldots, v_{m}$ are independent random vectors in $\mathbb{C}^{d}$ with support of size 2 such that $\sum_{i=1}^{m} \mathbb{E}\left[v_{i} v_{i}^{*}\right]=\mathbf{I}_{d}$ and $\mathbb{E}\left[\left\|v_{i}\right\|^{2}\right] \leq \varepsilon$ for all $i$, then

$$
\mathbb{P}\left(\left\|\sum_{i=1}^{m} v_{i} v_{i}^{*}\right\| \leq 1+2 \sqrt{\varepsilon(1-\varepsilon)}\right)>0
$$


Theorems 1.9 and 1.10 give the following quantitative bounds on the number of partitions $r$, see [2, Theorem 1.4].

Theorem 1.11 ([2]). Let $\varepsilon>0$ and suppose that $\left\{u_{i}\right\}_{i \in I}$ is a Bessel sequence in $\mathcal{H}$ with bound 1 that consists of vectors satisfying $\left\|u_{i}\right\|^{2} \geq \varepsilon$. Then there exists a universal constant $C>0$, such that $I$ can be partitioned into $r \leq \frac{C}{\varepsilon}$ subsets $I_{1}, \ldots, I_{r}$ such that every subfamily $\left\{u_{i}\right\}_{i \in I_{j}}, j=1, \ldots, r$, is a Riesz sequence in $\mathcal{H}$. Moreover, if $\varepsilon>3 / 4$, then $r=2$ works.

Applying Theorem 1.11 to exponential systems they obtained the following corollary [2, Corollary 6.16].

Theorem $1.12([2])$. There exists a universal constant $C>0$ such that for any subset $\mathcal{S} \subset \mathbb{T}$ with positive measure, the exponential system $E(\mathbb{Z})$ can be decomposed as a union of $r \leq C /|\mathcal{S}|$ Riesz sequences $E\left(\Lambda_{j}\right)$ in $L^{2}(\mathcal{S})$ for $j=1, \ldots, r$. Moreover, if $|\mathcal{S}|>3 / 4$, then $r=2$ works.

\section{Results}

The problem of attaining an explicit bound on the gap in the syndetic Riesz sequence was initially considered by Casazza and Tremain [5]. Their result [5, Theorem 6.17] states that every set $\mathcal{S}$ admits a syndetic Riesz sequence $E(\Lambda)$, with $\gamma(\Lambda) \leq c|\mathcal{S}|^{-8}$, but no proof was given.

The proof of Theorem 1.11 is based on defining random vectors appropriately so that with positive probability they facilitate a partition of the index set $I$ with desired properties. By modifying the definition of those random vectors we establish a quantitative bound for $\gamma(\Lambda)$. Our main result takes the form of a selection theorem for Bessel sequences with norms bounded from below.

Theorem 2.1. There exists a universal constant $C>0$ such that the following holds. Let $\varepsilon>0$ and suppose that $\left\{u_{i}\right\}_{i \in I}$ is a Bessel sequence in $\mathcal{H}$ with bound 1 and

$$
\left\|u_{i}\right\|^{2} \geq \varepsilon \quad \forall i \in I
$$

Then whenever $\left\{J_{k}\right\}_{k}$ is a collection of disjoint subsets of $I$ with $\# J_{k} \geq r=\left\lceil\frac{C}{\varepsilon}\right\rceil$, for all $k$, there exists a selector $J \subset \bigcup_{k} J_{k}$ satisfying

$$
\#\left(J \cap J_{k}\right)=1 \quad \forall k
$$

and such that $\left\{u_{i}\right\}_{i \in J}$ is a Riesz sequence in $\mathcal{H}$. Moreover, if $\varepsilon>\frac{3}{4}$, then the same conclusion holds with $r=2$. 
The proof of Theorem 2.1 is given in Section 3. Applying Theorem 2.1 to the normalized exponential system $\left\{\frac{1}{\sqrt{2 \pi}} e^{i n t}\right\}_{n \in \mathbb{Z}}$ in $L^{2}(\mathcal{S})$ with $J_{k}=[r k, r(k+1)) \cap \mathbb{Z}, k \in \mathbb{Z}$, where $r=$ $\lceil C /|\mathcal{S}|\rceil$, we obtain the following corollary.

Corollary 2.2. There exists a universal constant $C>0$ such that for any subset $\mathcal{S} \subset \mathbb{T}$ with positive measure, there exists a syndetic set $\Lambda \subset \mathbb{Z}$ with $\gamma(\Lambda) \leq C|\mathcal{S}|^{-1}$ so that $E(\Lambda)$ is a Riesz sequence in $L^{2}(\mathcal{S})$. Moreover, if $\frac{|\mathcal{S}|}{2 \pi}>3 / 4$, then such $\Lambda$ exists with $\gamma(\Lambda) \leq 3$.

Remark. Beyond improvements to the constant $C$, the bound in Corollary 2.2 is asymptotically sharp as $|\mathcal{S}| \rightarrow 0$. That is, we cannot expect to find a syndetic Riesz sequence with gaps of the order $|\mathcal{S}|^{\alpha}$ as $|\mathcal{S}| \rightarrow 0$ with $\alpha>-1$. This follows from Theorem 1.4 due to density considerations of the set $\Lambda$.

Theorem 2.1 can also be applied to the multidimensional setting. In this case we can deduce the existence of exponential Riesz sequences with syndetic-like properties.

Corollary 2.3. There exists a universal constant $C>0$ such that for any subset $\mathcal{S} \subset \mathbb{T}^{d}$ of positive measure, any d-dimensional rectangle $\mathcal{R}$ with $\# \mathcal{R} \geq C|\mathcal{S}|^{-1}$, and any partition $\mathbb{Z}^{d}=\bigcup \mathcal{R}_{k}$ into disjoint union of translated copies of $\mathcal{R}$, there exists a set $\Lambda \subset \mathbb{Z}^{d}$ such that

$$
\#\left(\Lambda \cap \mathcal{R}_{k}\right)=1 \quad \forall k
$$

and $E(\Lambda)$ is a Riesz sequence in $L^{2}(\mathcal{S})$. If $\mathcal{R}$ is taken to be a cube, then we obtain a set $\Lambda \subset \mathbb{Z}^{d}$ with

$$
\sup _{\mu \in \mathbb{Z}^{d}} \inf _{\lambda \in \Lambda}|\lambda-\mu| \leq C \sqrt{d}|\mathcal{S}|^{-1 / d}
$$

Note that Theorem 2.1 does not put any restrictions on the choice of sets $\left\{J_{k}\right\}_{k}$ beyond the size requirement $\# J_{k} \geq r$ for all $k$. Consequently, in Corollary 2.3 we may choose for $\left\{\mathcal{R}_{k}\right\}_{k}$ any family of disjoint subsets of $\mathbb{Z}^{d}$ satisfying $\# \mathcal{R}_{k} \geq r$, where $r=C /|\mathcal{S}|$. However, the most interesting case occurs when we take a cube $\mathcal{R}=[0, s)^{d} \cap \mathbb{Z}^{d}$ with side length $s=\left\lceil(C /|\mathcal{S}|)^{-1 / d}\right\rceil$ and the corresponding lattice partition

$$
\mathcal{R}_{k}=k+\mathcal{R}, \quad k \in s \mathbb{Z}^{d} .
$$

This choice yields a set $\Lambda \subset \mathbb{Z}^{d}$ satisfying the bound (4) such that $E(\Lambda)$ is a Riesz sequence in $L^{2}(\mathcal{S})$. Partitioning the lattice $\mathbb{Z}^{d}$ in a more complicated pattern we obtain the following corollary.

Corollary 2.4. There exists a universal constant $C>0$ such that for any subset $\mathcal{S} \subset \mathbb{T}^{d}$ of positive measure, there exists a set $\Lambda \subset \mathbb{Z}^{d}$ so that $E(\Lambda)$ is a Riesz sequence in $L^{2}(\mathcal{S})$ and $\Lambda$ is syndetic along any of its one dimensional sections. That $i s$, for any $j=1, \ldots, d$, and 
for any $\left(k_{1}, \ldots, \hat{k}_{j}, \ldots, k_{d}\right) \in \mathbb{Z}^{d-1}$ the set

$$
\Lambda_{j}\left(k_{1}, \ldots, \hat{k}_{j}, \ldots, k_{d}\right)=\left\{k_{j} \in \mathbb{Z}:\left(k_{1}, \ldots, k_{j}, \ldots, k_{d}\right) \in \Lambda\right\}
$$

is a syndetic subset of integers with gap satisfying

$$
\gamma\left(\Lambda_{j}\left(k_{1}, \ldots, \hat{k}_{j}, \ldots, k_{d}\right)\right) \leq C d|\mathcal{S}|^{-1} .
$$

The notation $\hat{k}_{j}$ in the vector $\left(k_{1}, \ldots, \hat{k}_{j}, \ldots, k_{d}\right)$ means that the term $k_{j}$ is missing.

Proof. Let $\mathcal{S} \subset \mathbb{T}^{d}$ of positive measure. We wish to apply Theorem 2.1 to the normalized exponential system $(2 \pi)^{-d / 2} E\left(\mathbb{Z}^{d}\right)$, which is a Parseval frame in $L^{2}(\mathcal{S})$, so we are only left to specify how $\left\{J_{k}\right\}$ should be chosen.

Let $r=\lceil C /|\mathcal{S}|\rceil$, where $C$ is the same constant as in Theorem 2.1. Define the $d$-dimensional cube

$$
Q_{r, d}=\{0, \ldots, r-1\}^{d} .
$$

We shall construct a partition $\left\{J_{k, x}\right\}_{(k, x) \in(r \mathbb{Z})^{d} \times Q_{r, d-1}}$ of $\mathbb{Z}^{d}$ as follows. For $k \in(r \mathbb{Z})^{d}$, let $j=j(k) \in\{1, \ldots, d\}$ be such that

$$
j \equiv \frac{k_{1}+\ldots+k_{d}}{r} \bmod d .
$$

Define

$$
J_{k, x}=\left\{\left(k_{1}+x_{1}, \ldots, k_{j-1}+x_{j-1}, k_{j}+x, k_{j+1}+x_{j}, \ldots, k_{d}+x_{d-1}\right): x \in\{0, \ldots, r-1\}\right\} .
$$

Hence, for a fixed $k \in(r \mathbb{Z})^{d}$, the family $\left\{J_{k, x}\right\}_{x \in Q_{r, d-1}}$ is a partition of a cube $k+Q_{r, d}$ into line segments of length $r$ parallel to the $j$ axis. Now, the direction $j$ cycles according to (6) as a function of $k \in(r \mathbb{Z})^{d}$. In particular, for a fixed $j_{0}=1, \ldots, d$, cubes of the form $k+r\left(n d+j_{0}-j\right) e_{j_{0}}+Q_{r, d}$, where $n \in \mathbb{Z}, j$ is given by (6) and $e_{j_{0}}$ is the $j_{0}$ 'th coordinate vector, are partitioned into line segments parallel to the $j_{0}$ axis. Since Theorem 2.1 guarantees that the selection set $\Lambda$ contains one element from each such line segment, we deduce that $\Lambda$ is syndetic along every one dimensional section in the direction of $e_{j}$ with gap bounded by $2 d r$. This yields the bound (5). Moreover, $E(\Lambda)$ is a Riesz sequence in $L^{2}(\mathcal{S})$ by Theorem 2.1.

In Section 3 we discuss the proof of Theorem 2.1 in detail. Its proof uses Theorems 1.9 and 1.10, which involve probabilistic elements. This, in essence, makes the task of extracting a deterministic construction of the set $\Lambda$ extremely difficult, and the authors do not know how this might be done for an arbitrary set. Furthermore, while Corollary 2.2 is sharp in the sense that asymptotically as $|\mathcal{S}| \rightarrow 0$, we are also interested in another kind of sharpness which may occur as $\frac{|\mathcal{S}|}{2 \pi} \rightarrow 1$. That is, for sets of large measure we wish to remove from $\mathbb{Z}$ a uniformly separated set with gaps size not smaller than $C\left|\mathcal{S}^{c}\right|^{-1}$. It turns out that in the 
case where $\mathcal{S}$ is an open set a deterministic construction does exist and can be chosen to satisfy these properties.

Theorem 2.5. There exists a universal constant $C>0$ such that for any open subset $\mathcal{S} \subset \mathbb{T}$, one can construct a syndetic set $\Lambda \subset \mathbb{Z}$ with gaps between consecutive elements taking exactly two values $\{1, d\}$, where $d=d(\mathcal{S}) \leq \frac{C}{|\mathcal{S}|}$, and so that $E(\Lambda)$ is a Riesz sequence in $L^{2}(\mathcal{S})$. Moreover, if $\frac{|\mathcal{S}|}{2 \pi}>\frac{1}{2}$, then $\Lambda$ may be chosen so that $\Lambda^{c}=\mathbb{Z} \backslash \Lambda$ is uniformly separated and satisfies

$$
\inf _{\lambda, \mu \in \Lambda^{c}, \lambda \neq \mu}|\lambda-\mu| \geq \frac{C}{\left|\mathcal{S}^{c}\right|}
$$

It should be noted that the sheer existence of syndetic Riesz sequences for open sets is trivial since any open set admits an infinite arithmetic progression as a Riesz sequence. However, Theorem 2.5 ensures the existence of such systems together with an effective estimate on the gap. The proof of Theorem 2.5 is based on a totally different approach than that of Theorem 2.1 and uses simple quasicrystals. This will be discussed in Section 4.

\section{The Syndetic RIESz SEQUence BOUnd - General CASE}

In this section we prove our main result Theorem 2.1. Theorem 1.9 gives rise to a selecting mechanism which allows, from every Parseval frame with certain norms, a probabilistic selection of a Bessel subsystem with smaller bound. Note that by the Schur-Horn theorem we may apply Theorem 1.9 to Bessel sequences with bound 1 by completing them to a Parseval frame. Indeed, we have the following lemma from [2].

Lemma 3.1. Let $\mathcal{H}$ be an infinite dimensional Hilbert space, $M \in \mathbb{N}$ and $\delta \in(0,1)$. Suppose $\left\{u_{i}\right\}_{i=1}^{M} \subset \mathcal{H}$ is a Bessel sequence with Bessel bound 1 and $\left\|u_{i}\right\|^{2} \geq \delta$ for all $i$, or $\left\|u_{i}\right\|^{2} \leq \delta$ for all $i$. Then for every large enough $K \in \mathbb{N}$, there exist vectors $\varphi_{1}, \ldots, \varphi_{K} \in \mathcal{H}$ with $\left\|\varphi_{i}\right\|^{2} \geq \delta$ for all $i$, or $\left\|\varphi_{i}\right\|^{2} \leq \delta$ for all $i$, respectively, such that $\left\{u_{i}\right\}_{i=1}^{M} \cup\left\{\varphi_{i}\right\}_{i=1}^{K}$ is a Parseval frame for its linear span.

Proof. The case $\left\|u_{i}\right\|^{2} \geq \delta$ of Lemma 3.1 follows from the Schur-Horn theorem and it can be found in [2, Corollary 6.6]. However, the case $\left\|u_{i}\right\|^{2} \leq \delta$ is much simpler as it does not require the use of the Schur-Horn theorem. Indeed, consider the frame operator $S$ which corresponds to $\left\{u_{i}\right\}_{i=1}^{M}$. It is well known that $S$ is a self-adjoint operator (see [18]), and so denoting its non-zero eigenvalues $1 \geq \lambda_{n} \geq \ldots \geq \lambda_{1}>0$ and corresponding orthonormal eigenvectors $v_{1}, \ldots, v_{n}$ we may write

$$
S=\sum_{i=1}^{n} \lambda_{i}\left\langle\cdot, v_{i}\right\rangle v_{i} .
$$


Then we choose a number $m$ such that $\frac{1-\lambda_{n}}{m}<\delta$, and for each $i \in[n]$ we take $m$ copies of the vector $\sqrt{\frac{1-\lambda_{i}}{m}} v_{i}$. By the choice of $m$

$$
\left\|\sqrt{\frac{1-\lambda_{i}}{m}} v_{i}\right\|^{2} \leq \delta \quad \forall i \in[n]
$$

and

$$
S+\sum_{j=1}^{m} \sum_{i=1}^{n} \frac{1-\lambda_{i}}{m}\left\langle\cdot, v_{i}\right\rangle v_{i}=\mathbf{I}_{H},
$$

where $H=\operatorname{span}\left\{u_{1}, \ldots, u_{M}\right\}=\operatorname{span}\left\{v_{1}, \ldots, v_{n}\right\}$. Here and below $\mathbf{I}_{\mathcal{H}}$ denotes the identity map on $\mathcal{H}$.

Recall that by Naimark's dilation theorem [6, Proposition 1.1] a system $\left\{u_{i}\right\}_{i \in I}$ is a Parseval frame for $H$ if and only if there exists a containing space $H \subseteq \mathcal{H}$ and an orthonormal basis $\left\{e_{i}\right\}_{i \in I}$ for $\mathcal{H}$ such that $P e_{i}=u_{i}$ for all $i$. Here $P: \mathcal{H} \rightarrow \mathcal{H}$ is the orthogonal projection onto $H$. It turns out that by considering a certain dual system, called Naimark's complement, we may choose a subsystem of $\left\{u_{i}\right\}_{i \in I}$ which is a Riesz sequence, see [2, Proposition 5.4].

Lemma $3.2([2])$. Let $P: \mathcal{H} \rightarrow \mathcal{H}$ be the orthogonal projection onto a closed subspace $H \subset \mathcal{H}$, and let $\left\{e_{i}\right\}_{i \in I}$ be an orthonormal basis for $\mathcal{H}$. Then for any subset $J \subset I$ and $\delta>0$ the following are equivalent:

(1) $\left\{P e_{i}\right\}_{i \in J}$ is a Bessel sequence with bound $1-\delta$.

(2) $\left\{\left(\mathbf{I}_{\mathcal{H}}-P\right) e_{i}\right\}_{i \in J}$ is a Riesz sequence with lower Riesz bound $\delta$.

The following theorem demonstrates how Theorem 1.9 can be applied in order to find a selector.

Theorem 3.3. Let $r, M \in \mathbb{N}$ and $\delta>0$. Suppose that $\left\{u_{i}\right\}_{i=1}^{M} \subset \mathcal{H}$ is a Bessel sequence with bound 1 and $\left\|u_{i}\right\|^{2} \leq \delta$ for all $i$. Then for every collection of disjoint subsets $J_{1}, \ldots, J_{n} \subset[M]$ with $\# J_{k} \geq r$ for all $k$, there exists a subset $J \subset[M]$ such that

$$
\#\left(J \cap J_{k}\right)=1 \quad \forall k \in[n]
$$

and the system of vectors $\left\{u_{i}\right\}_{i \in J}$ is a Bessel sequence with bound

$$
\left(\frac{1}{\sqrt{r}}+\sqrt{\delta}\right)^{2}
$$

Proof. Without loss of generality we can assume that $\# J_{k}=r$ for all $k$. Consider the system of vectors $\left\{u_{i}\right\}_{i \in \cup_{k=1}^{n} J_{k}}$ and note that it is Bessel sequence with bound 1. By Lemma 3.1 we can find finitely many vectors $\varphi_{1}, \ldots, \varphi_{r K}$ with $\left\|\varphi_{i}\right\|^{2} \leq \delta$ for all $i$ and such that $\left\{u_{i}\right\}_{i \in \bigcup_{k=1}^{n} J_{k}} \cup\left\{\varphi_{i}\right\}_{i=1}^{r K}$ is a Parseval frame for some finite dimensional subspace $H \subset \mathcal{H}$. 
Define the independent random vectors $v_{1}, \ldots, v_{n+K}$ as follows. For $k=1, \ldots, n$ we let the vector $v_{k}$ to take values $\sqrt{r} u_{i}$ for any $i \in J_{k}$ with equal probability $\frac{1}{r}$. For $k=n+1, \ldots n+K$, we set

$$
\mathbb{P}\left(v_{k}=\sqrt{r} \varphi_{r(k-(n+1))+1}\right)=1 / r, \ldots, \mathbb{P}\left(v_{k}=\sqrt{r} \varphi_{r(k-n)}\right)=1 / r
$$

Note that

$$
\mathbb{E}\left\|v_{k}\right\|^{2} \leq r \delta \quad \forall k \in[n+K]
$$

as well as

$$
\sum_{k=1}^{n+K} \mathbb{E}\left(v_{k} v_{k}^{*}\right)=\sum_{i \in \bigcup_{k=1}^{n} J_{k}} u_{i} u_{i}^{*}+\sum_{i=1}^{r K} \varphi_{i} \varphi_{i}^{*}=\mathbf{I}_{H}
$$

It follows from Theorem 1.9 that

$$
\mathbb{P}\left(\left\|\sum_{k=1}^{n+K} v_{k} v_{k}^{*}\right\| \leq(1+\sqrt{r \delta})^{2}\right)>0 .
$$

This implies the existence of a set $J \subset[M]$ satisfying (8) such that

$$
\left\|\sum_{i \in J} u_{i} u_{i}^{*}\right\| \leq\left(\frac{1}{\sqrt{r}}+\sqrt{\delta}\right)^{2}
$$

as required.

In the case the vectors in our systems are short we can obtain a variant of Theorem 3.3. Theorem 3.4 follows from Theorem 1.10 in a similar fashion as Theorem 3.3 follows from Theorem 1.9, see [2, Theorem 6.3].

Theorem 3.4. Let $M \in \mathbb{N}$ and $\delta_{0} \in(0,1 / 4)$. Suppose that $\left\{u_{i}\right\}_{i=1}^{M} \subset \mathcal{H}$ is a Bessel sequence with Bessel bound 1 and $\left\|u_{i}\right\|^{2} \leq \delta_{0}$ for all $i$. Then for every collection of disjoint subsets $J_{1}, \ldots, J_{n} \subset[M]$ with $\# J_{k}=2$ for all $k$, there exists a subset $J \subset[M]$ such that $\#\left(J \cap J_{k}\right)=$ 1 for all $k \in[n]$, and the system of vectors $\left\{u_{i}\right\}_{i \in J}$ is a Bessel sequence with bound $1-\varepsilon_{0}$, where $\varepsilon_{0}=\frac{1}{2}-\sqrt{2 \delta_{0}\left(1-2 \delta_{0}\right)}$.

Throughout this section we fix some value of $\delta_{0} \in(0,1 / 4)$ and the corresponding $\varepsilon_{0}=$ $\frac{1}{2}-\sqrt{2 \delta_{0}\left(1-2 \delta_{0}\right)}$. As a corollary we obtain a way to extract a syndetic Riesz sequence from a given Bessel sequence, provided that the vectors in the system are not too short.

Corollary 3.5. Let $M \in \mathbb{N}$ and $\delta_{0} \in(0,1 / 4)$. Suppose that $\left\{u_{i}\right\}_{i=1}^{M} \subset \mathcal{H}$ is a Bessel sequence with Bessel bound $B$ and $\left\|u_{i}\right\|^{2} \geq B\left(1-\delta_{0}\right)$ for all $i$. Then for every collection of disjoint subsets $J_{1}, \ldots, J_{n} \subset[M]$ with \# $J_{k}=2$ for all $k$, there exists a subset $J \subset[M]$ such that $\#\left(J \cap J_{k}\right)=1$ for all $k \in[n]$ and the system of vectors $\left\{u_{i}\right\}_{i \in J}$ is a Riesz sequence with lower Riesz bound $B \varepsilon_{0}$. 
Proof. Without loss of generality we may assume $B=1$. Let $J_{1}, \ldots, J_{n} \subset[M]$ be a disjoint collection of subsets with \# $J_{k}=2$ for all $k$. Consider the system of vectors $\left\{u_{i}\right\}_{i \in \bigcup_{k=1}^{n} J_{k}}$ and note that it is Bessel sequence with bound 1. Then applying Lemma 3.1 to $\left\{u_{i}\right\}_{i \in \bigcup_{k=1}^{n} J_{k}}$, we find finitely many vectors $\varphi_{1}, \ldots, \varphi_{2 K}$ with $\left\|\varphi_{i}\right\|^{2} \geq 1-\delta_{0}$ for all $i$ and such that $\left\{u_{i}\right\}_{i \in \bigcup_{k=1}^{n} J_{k}} \cup$ $\left\{\varphi_{i}\right\}_{i=1}^{2 K}$ is a Parseval frame for its linear span, denoted by $H$.

For convenience we rename our vectors as $\left\{g_{i}\right\}_{i=1}^{2(n+k)}$ as follows. We write $J_{k}=\left\{j_{1}^{k}, j_{2}^{k}\right\}$ and define

$$
g_{2 k-1}=u_{j_{1}^{k}}, \quad g_{2 k}=u_{j_{2}^{k}}, \quad k=1, \ldots, n,
$$

and $\left\{g_{i}\right\}_{i=2 n+1}^{2(n+K)}=\left\{\varphi_{i}\right\}_{i=1}^{2 K}$. By Naimark's dilation theorem there exists a $2(n+K)$ dimensional space $\mathcal{K} \supseteq H$ with a corresponding orthonormal basis $\left\{e_{i}\right\}_{i=1}^{2(n+K)}$ and an orthogonal projection $P: \mathcal{K} \rightarrow \mathcal{K}$ onto $H$ such that

$$
P e_{i}=g_{i} \quad \text { for } i=1,2, \ldots, 2(n+K)
$$

Then, setting $f_{i}=\left(\mathbf{I}_{\mathcal{K}}-P\right) e_{i}$ for $i=1, \ldots, 2(n+K)$, it follows that $\left\{f_{i}\right\}$ is a Parseval frame for its linear span with $\left\|f_{i}\right\|^{2} \leq \delta_{0}$. By Theorem 3.4 applied to the collection $J_{k}^{\prime}=$ $\{2 k-1,2 k\}, k=1, \ldots, n+K$, there is a subset $J^{\prime} \subset[2(n+K)]$ such that $\#\left(J^{\prime} \cap J_{k}^{\prime}\right)=1$ for all $k \in[n+K]$ and $\left\{f_{i}\right\}_{i \in J^{\prime}}$ is a Bessel sequence with bound $1-\varepsilon_{0}$. By Lemma 3.2 $\left\{g_{i}\right\}_{i \in J^{\prime}}$ is a Riesz sequence with lower bound $\varepsilon_{0}$. Hence, there exists $J \subset[M]$ such that $\#\left(J \cap J_{k}\right)=1$ for all $k \in[n]$ and the system of vectors $\left\{u_{i}\right\}_{i \in J}$ is a Riesz sequence with lower bound $\varepsilon_{0}$.

Combining Theorem 3.3 and Corollary 3.5 we get a finite dimensional version of Theorem 2.1 .

Theorem 3.6. Let $\varepsilon>0$ and $M \in \mathbb{N}$. Suppose that $\left\{u_{i}\right\}_{i=1}^{M} \subset \mathcal{H}$ is a Bessel sequence with Bessel bound 1 and $\left\|u_{i}\right\|^{2} \geq \varepsilon$ for all $i$. Then there exists $r=O(1 / \varepsilon)$, independent of $M$, such that for every collection of disjoint subsets $J_{1}, \ldots, J_{n} \subset[M]$ with $\# J_{k} \geq r$ for all $k$, there exists a subset $J \subset[M]$ such that $\#\left(J \cap J_{k}\right)=1$ for all $k \in[n]$ and $\left\{u_{i}\right\}_{i \in J}$ is a Riesz sequence with lower Riesz bound $\varepsilon \varepsilon_{0}$. Moreover, if $\varepsilon>3 / 4$ then the same conclusion holds with $r=2$.

Proof. Without loss of generality we may assume $\left\|u_{i}\right\|^{2}=\varepsilon$ for all $i$; otherwise replace $u_{i}$ with $\sqrt{\varepsilon} /\left\|u_{i}\right\| u_{i}$. Let $C$ be an absolute constant to be specified later and set $r=2\left\lceil\frac{C}{\varepsilon}\right\rceil$. Let $J_{1}, \ldots, J_{n} \subset[M]$ be a collection of disjoint subsets with $\# J_{k} \geq r$ for all $k$.

Let $\tilde{r}=\frac{r}{2}$. For every $k \in[n]$ choose a partition $J_{k}=\Lambda_{2 k-1} \cup \Lambda_{2 k}$ such that $\# \Lambda_{\ell} \geq \tilde{r}$ for all $\ell \in[2 n]$. By Theorem 3.3 we may find a subset $J \subset[2 n]$ such that $\#\left(J \cap \Lambda_{\ell}\right)=1$ for all 
$\ell \in[2 n]$, and the system of vectors $\left\{u_{i}\right\}_{i \in J}$ is a Bessel sequence with bound

$$
\left(\frac{1}{\sqrt{\tilde{r}}}+\sqrt{\varepsilon}\right)^{2} \text {. }
$$

Then we partition $J=\bigcup_{m=1}^{n} P_{m}$ into pairs $P_{m}=\left\{j_{2 m-1}, j_{2 m}\right\}$ so that $j_{\ell} \in J \cap \Lambda_{\ell}$ for all $\ell \in[2 n]$. Next we wish to apply Corollary 3.5 to $\left\{u_{i}\right\}_{i \in J}$, and we may do that if for some $\delta_{0} \in(0,1 / 4)$ we have

$$
\varepsilon \geq\left(\frac{1}{\sqrt{\tilde{r}}}+\sqrt{\varepsilon}\right)^{2}\left(1-\delta_{0}\right) .
$$

By a simple calculation this holds true whenever $\tilde{r} \geq \frac{C}{\varepsilon}$, with $C=9\left(\frac{1-\delta_{0}}{\delta_{0}}\right)^{2}$, see the proof [2, Theorem 6.7]. By Corollary 3.5 applied to the collection $P_{1}, \ldots, P_{n}$, there exists a subset $J^{\prime} \subset[n]$ such that $\#\left(J^{\prime} \cap P_{m}\right)=1$ for all $m \in[n]$, and the system of vectors $\left\{u_{i}\right\}_{i \in J^{\prime}}$ is a Riesz sequence with lower bound $\left(\frac{1}{\sqrt{\tilde{r}}}+\sqrt{\varepsilon}\right)^{2} \varepsilon_{0} \geq \varepsilon \varepsilon_{0}$. For the moreover part we apply Corollary 3.5 directly, setting $\delta_{0}=1-\varepsilon$.

In order to prove Theorem 2.1 we will require the following lemma.

Lemma 3.7. Let $\left\{J_{k}\right\}_{k}$ be a collection of disjoint subsets of $I$. Assume for every $n \in \mathbb{N}$ we have a subset $I_{n} \subset \bigcup_{k=1}^{n} J_{k}$ such that

$$
\#\left(I_{n} \cap J_{k}\right)=1 \quad \forall k \in[n] .
$$

Then, there exists a subset $I_{\infty} \subset I$ and an increasing sequence $\left\{n_{j}\right\}$ such that

$$
I_{n_{j}} \cap\left(\bigcup_{k=1}^{j} J_{k}\right)=I_{\infty} \cap\left(\bigcup_{k=1}^{j} J_{k}\right) \quad \forall j .
$$

In particular, we have

$$
\#\left(I_{\infty} \cap J_{k}\right)=1 \quad \forall k \in \mathbb{N}
$$

Lemma 3.7 is proved by a combination of a diagonal argument with the pigeonhole principle. For a similar result involving partitions, see [3, Proposition 2.1].

Proof of Theorem 2.1. Let $r=r(\varepsilon)$ be as in Theorem 3.6 and $\left\{J_{k}\right\}_{k}$ a collection of disjoint subsets of $I$ with $\# J_{k} \geq r$, for all $k$.

For every $n \in \mathbb{N}$ apply Theorem 3.6 to the collection $J_{1}, \ldots, J_{n}$. This gives a subset $I_{n} \subset \bigcup_{k=1}^{n} J_{k}$ satisfying (9) and such that the system of vectors $\left\{u_{i}\right\}_{i \in I_{n}}$ is a Riesz sequence in $\mathcal{H}$ with lower bound $\varepsilon \varepsilon_{0}$. By Lemma 3.7 there exist an increasing sequence $\left\{n_{j}\right\}$ and a subset $I_{\infty} \subset I$ satisfying (10). We claim that $\left\{u_{i}\right\}_{i \in I_{\infty}}$ is a Riesz sequence in $\mathcal{H}$ with the same lower bound. This is true since every finite subsystem is contained in $\left\{u_{i}\right\}_{i \in I_{n_{j}}}$, given that $j$ is large enough. 
It is worth to mention an extension of Theorem 2.1 in which we are interested in finding a syndetic Riesz sequence with tight bounds. This problem was known as the $R_{\varepsilon}$ conjecture.

Theorem 3.8. There exists a universal constant $C>0$ such that following holds. Let $\left\{u_{i}\right\}_{i \in I}$ be a unit norm Bessel sequence in $\mathcal{H}$ with bound B. For any $\varepsilon>0$ and any collection of disjoint subsets of $I,\left\{J_{k}\right\}_{k}$ satisfying $\# J_{k} \geq r=\left\lceil C \frac{B}{\varepsilon^{4}}\right\rceil$ for all $k$, there exists a selector $J \subset \bigcup_{k} J_{k}$ satisfying

$$
\#\left(J \cap J_{k}\right)=1 \quad \forall k
$$

and such that $\left\{u_{i}\right\}_{i \in J}$ is a Riesz sequence in $\mathcal{H}$ with bounds $1-\varepsilon$ and $1+\varepsilon$.

Remark. Theorem 3.8, when applied to exponential systems, provides an improvement of [5, Theorem 6.4]. A recent multi-paving result of Ravichandran and Srivastava [20] suggests that this result might hold with $r=O\left(\frac{B}{\varepsilon^{2}}\right)$.

We will give an outline of the proof of Theorem 3.8. This will require the following lemma, which appeared in [21]. A slightly more general formulation, given below, is taken from [2, Lemma 6.13].

Lemma $3.9([2])$. Suppose $\left\{u_{i}\right\}_{i \in I}$ is a Riesz basis in $\mathcal{H}$, and let $\left\{u_{i}^{*}\right\}_{i \in I}$ be its unique biorthogonal Riesz basis. Then for any subset $J \subset I$, the Riesz sequence bounds of $\left\{u_{i}\right\}_{i \in J}$ are $A$ and $B$ if and only if the Riesz sequence bounds of $\left\{u_{i}^{*}\right\}_{i \in J}$ are $1 / B$ and $1 / A$.

The proof of Theorem 3.8 proceeds as follows. By Lemma 3.7 it suffices to consider a finite system $\left\{u_{i}\right\}_{i=1}^{M}$ that is a unit norm Bessel sequence with upper bound $B$. Fix a collection $J_{1}, \ldots, J_{n} \subset[M]$ with $\# J_{k} \geq r$ for all $k$. Then the subsystem we are looking for is obtained in 3 steps. In the first step, using Theorem 3.6, we find a subsystem $\left\{u_{i}\right\}_{i \in J}$ which is a Riesz sequence with lower Riesz bound $\varepsilon_{0}$ and upper Riesz bound $\frac{1}{1-\delta_{0}}$, and $\#\left(J \cap J_{k}\right) \geq\left\lceil\frac{C}{\varepsilon^{2}}\right\rceil^{2}$ for all $k$. In the second step we apply Theorem 3.3 to $\left\{u_{i}\right\}_{i \in J}$ and extract from it another subsystem $\left\{u_{i}\right\}_{i \in J^{\prime}}, J^{\prime} \subset J$, having its upper Riesz bound reduced to $1+\varepsilon$. As a consequence we get $\#\left(J^{\prime} \cap J_{k}\right) \geq\left\lceil\frac{C}{\varepsilon^{2}}\right\rceil$ for all $k$. In the last step we move to the dual system $\left\{u_{i}^{*}\right\}_{i \in J^{\prime}}$ which, by Lemma 3.9, has upper Riesz bound $\frac{1}{\varepsilon_{0}}$. Apply to it Theorem 3.3 and get a subsystem $\left\{u_{i}^{*}\right\}_{i \in J^{\prime \prime}}, J^{\prime \prime} \subset J^{\prime}$ with upper Riesz bound reduced to $1+\varepsilon$, and $\#\left(J^{\prime \prime} \cap J_{k}\right)=1$ for all $k$. Now $\left\{u_{i}\right\}_{i \in J^{\prime \prime}}$ is the desired subsystem, keeping in mind that $\frac{1}{1+\varepsilon} \geq 1-\varepsilon$.

\section{Open Sets}

In this section we prove Theorem 2.5, namely we present a construction of a syndetic exponential Riesz sequence which is asymptotically sharp in both senses described in Section 2. This will be done applying a particular case of Fourier quasicrystals. We begin with some background. 
4.1. Universal Riesz sequences. In their paper [17] Olevskii and Ulanovskii asked whether there exists a set $\Lambda$ such that $E(\Lambda)$ is a Riesz sequence in $L^{2}(\mathcal{S})$ for all sets $\mathcal{S} \subset \mathbb{T}$ with large measure. As it turns out, the answer depends considerably on the spectrum $\mathcal{S}$. It is positive if we restrict ourselves to open spectra.

Theorem 4.1 ([17, Theorem 5]). For every $d \in(0,1)$, there is a universal Riesz sequence, i.e. a set $\Lambda \subset \mathbb{R}$ with $D(\Lambda)=d$ such that $E(\Lambda)$ is a Riesz sequence in $L^{2}(\mathcal{S})$ for every open set $\mathcal{S} \subset \mathbb{T}$ with $\frac{|\mathcal{S}|}{2 \pi}>d$.

Note that the set $\Lambda$ constructed in the proof of Theorem 4.1 is a specially chosen perturbation of the lattice $1 / d \mathbb{Z}$. On the other hand, considering all measurable sets the answer is negative.

Theorem 4.2 ([17, Theorem 4]). Let $d \in(0,1)$. Then for every $\varepsilon \in(0,1)$ and $\Lambda \subset \mathbb{R}$ with $D(\Lambda)=d$, there exists a set $\mathcal{S} \subset \mathbb{T}$ with $\frac{|\mathcal{S}|}{2 \pi}>1-\varepsilon$ such that $E(\Lambda)$ is not a Riesz sequence in $L^{2}(\mathcal{S})$.

4.2. Quasicrystals. The mathematical model of quasicrystals, introduced by Meyer in 1972 (see [15]), is a construction based on the "cut and project" process which produces what is known as Meyer's "model set". In the periodic case a model set takes the following form. We use the notation from [8].

Definition 4.3. Let $\alpha$ be an irrational number, and $I=[a, b) \subset[0,1]$. The (simple) quasicrystal corresponding to $\alpha$ and $I$ is

$$
\Lambda(\alpha, I)=\{n \in \mathbb{Z} \mid\{\alpha n\} \in I\}
$$

where $\{x\}$ is the fractional part of the real number $x$.

The set $\Lambda(\alpha, I)$ has many interesting and important properties, see, for instance, [15] and [16]. A basic property which follows from the fact that the sequence $\{\alpha n\}$ is equidistributed in $[0,1]$ is that the corresponding simple quasicrystal had uniform density $D(\Lambda(\alpha, I))=$ $|I|$. One key property of the exponential system $E(\Lambda(\alpha, I))$ that will be useful for us is universality. Matei and Meyer showed that simple quasicrystals are an alternative example of universal Riesz sequences with the additional property that they are contained in the integers rather than in the real line (see [12]-[14]). We will require a version of Meyer's duality principle, we use the formulation given in [8, Lemma 2.1].

Lemma $4.4([8])$. Let $U \subset[0,1]$ be a semi-closed multiband set, and $V \subset[0,1]$ a multiband set. Then:

(1) If $E(2 \pi \Lambda(\alpha, V))$ is a frame in $L^{2}(U)$, then $E(-2 \pi \Lambda(\alpha, U))$ is a Riesz sequence in $L^{2}(V)$. 
(2) If $E(2 \pi \Lambda(\alpha, V))$ is a Riesz sequence in $L^{2}(U)$, then $E(-2 \pi \Lambda(\alpha, U))$ is a frame in $L^{2}(V)$.

Here, a set is called multiband if it is a union of finitely many intervals. A multiband set is called semi-closed if its indicator function is continuous either from the left or from the right. Combining Lemma 4.4 and Beurling's sufficient condition for frames [18, Theorem 3.33] together with a rescaling argument gives the following universality result of Matei and Meyer [13], see [18, Section 6.6.5].

Theorem 4.5 ([13]). The system $E(\Lambda(\alpha, I))$ is a universal Riesz sequence, i.e., $E(\Lambda(\alpha, I))$ is a Riesz sequence in $L^{2}(\mathcal{S})$ for every open set $\mathcal{S} \subset \mathbb{T}$ with $\frac{|\mathcal{S}|}{2 \pi}>|I|$.

Proof of Theorem 2.5. Let $n \geq 2$ be an integer such that $\frac{1}{n}<\frac{|S|}{2 \pi} \leq \frac{1}{n-1}$. Fix an irrational $\frac{1}{n}<a<\frac{1}{n+1}$ such that $\frac{|\mathcal{S}|}{2 \pi}>a$. Set the irrational $\alpha=\frac{1-a}{n-1}$ and define $I=[0, a)$. Now consider the simple quasicrystal

$$
\Lambda(\alpha, I)=\left\{\lambda_{j}: j \in \mathbb{Z}\right\} \quad \text { where } \lambda_{j}<\lambda_{j+1} \text { for all } j \in \mathbb{Z} .
$$

By Theorem $4.5 E(\Lambda(\alpha, I))$ is a Riesz sequence in $L^{2}(\mathcal{S})$ since $\frac{|\mathcal{S}|}{2 \pi}>|I|=a$. Moreover, if for some $k \in \mathbb{Z}$

$$
0 \leq\{\alpha k\}<a \quad \text { and } \quad a \leq\{\alpha(k+1)\}<1
$$

then by the choice of $\alpha$ we have

$$
a \leq\{\alpha(k+j)\}<1 \quad \text { for } j \in\{2, \ldots, n-1\} .
$$

Since $\alpha<a$ we also have

$$
\{\alpha(k+n)\} \in I .
$$

This implies that

$$
\lambda_{j+1}-\lambda_{j} \in\{1, n\} \quad \text { for all } j \in \mathbb{Z} .
$$

For the moreover part we let $n \geq 2$ be such that $1-\frac{1}{n}<\frac{|S|}{2 \pi} \leq 1-\frac{1}{n+1}$. Fix an irrational $\frac{1}{n+1}<a<\frac{1}{n}$ such that $\frac{|S|}{2 \pi}>1-a$. We set $\alpha=\frac{1-a}{n-1}$ and $I=[0, a)$ as above. Thus, by Theorem $4.5 E\left(\Lambda\left(\alpha, I^{c}\right)\right)$ is a Riesz sequence in $L^{2}(\mathcal{S})$, where $I^{c}=[a, 1)$. Since $\alpha>a$, if $\{\alpha k\} \in I$ for some $k \in I$, then $\{\alpha(k+1)\} \in I^{c}$ and consequently we have

$$
\{\alpha(k+j)\} \in I^{c} \quad \text { for } j \in\{1, \ldots, n-1\} .
$$

Hence, the difference between consecutive elements in $\Lambda(\alpha, I)$ is bounded below by $n$. Since

$$
\Lambda\left(\alpha, I^{c}\right)=\mathbb{Z} \backslash \Lambda(\alpha, I)
$$

we deduce that $\Lambda\left(\alpha, I^{c}\right)$ satisfies $(7)$. 
There are still a few open problems left with regards to the subject of syndetic Riesz sequences. Below we give a couple of them.

Open problem 1. By Theorem 4.2, $E(\Lambda(\alpha, I))$ is not a Riesz sequence in $L^{2}(\mathcal{S})$ for some non-open set $\mathcal{S} \subset \mathbb{T}$ with $\frac{|\mathcal{S}|}{2 \pi}>|I|$. Does there exist $\mathcal{S}$ with empty interior for which $E(\Lambda(\alpha, I))$ is a Riesz sequence? Otherwise prove that such set does not exist.

Open problem 2. Does the moreover part of Theorem 2.5 hold in the general case? That is, an arbitrary measurable set $\mathcal{S} \subset \mathbb{T}$ with $\frac{|\mathcal{S}|}{2 \pi}>\frac{1}{2}$ admits an exponential Riesz sequence $E(\Lambda)$ such that $\Lambda^{c}=\mathbb{Z} \backslash \Lambda$ satisfies

$$
\inf _{\lambda, \mu \in \Lambda^{c}, \lambda \neq \mu}|\lambda-\mu| \geq \frac{C}{\left|\mathcal{S}^{c}\right|} .
$$

\section{REFERENCES}

[1] J. Bourgain and L. Tzafriri, Invertibility of "large" submatrices with applications to the geometry of Banach spaces and harmonic analysis, Israel J. Math. 57 (1987), no. 3, $137-224$.

[2] M. Bownik, P. Casazza, A. Marcus, and D. Speegle, Improved bounds in Weaver and Feichtinger conjectures, J. Reine Angew. Math. (to appear).

[3] P. Casazza, O. Christensen, A. Lindner, and R. Vershynin, Frames and the Feichtinger conjecture, Proc. Amer. Math. Soc. 133 (2005), no. 4, 1025-1033.

[4] P. Casazza and J. Tremain, The Kadison-Singer problem in mathematics and engineering, Proc. Nat. Acad. Sci. USA 103 (2006), no. 7, 2032-2039.

[5] P. Casazza and J. Tremain, Consequences of the Marcus/Spielman/Srivastava solution of the Kadison-Singer problem, New trends in applied harmonic analysis, 191-213, Appl. Numer. Harmon. Anal., Birkhaüser/Springer, Cham, 2016.

[6] D. Han and D. Larson, Frames, bases and group representations, Mem. Amer. Math. Soc. 147 (2000), no. 697, x+94 pp.

[7] J.-P. Kahane, Sur les fonctions moyenne-périodiques bornées, Ann. Inst. Fourier 7 (1957), 293-314.

[8] G. Kozma and N. Lev, Exponential Riesz bases, discrepancy of irrational rotations and BMO, J. Fourier Anal. Appl. 17 (2011), no. 5, 879-898.

[9] H. Landau, Necessary density conditions for sampling and interpolation of certain entire functions, Acta Math. 117 (1967), no. 1, 37-52.

[10] W. Lawton, Minimal sequences and the Kadison-Singer problem, Bull. Malays. Math. Sci. Soc. 33 (2010), no. 2, 169-176.

[11] A. Marcus, D.A. Spielman, and N. Srivastava, Interlacing Families II: Mixed Characteristic Polynomials and the Kadison-Singer Problem, Ann. of Math. 182 (2015), no. $1,327-350$. 
[12] B. Matei and Y. Meyer, Quasicrystals are sets of stable sampling, C. R. Math. Acad. Sci. Paris 346 (2008), no. 23-24, 1235-1238.

[13] B. Matei and Y. Meyer, A variant of compressed sensing, Rev. Mat. Iberoamericana 25 (2009), no. 2, 669-692.

[14] B. Matei and Y. Meyer, Simple quasicrystals are sets of stable sampling, Complex Var. Elliptic Equ. 55 (2010), no. 8-10, 947-964.

[15] Y. Meyer, Algebraic numbers and harmonic analysis, North-Holland Mathematical Library, Vol. 2. North-Holland Publishing Co., Amsterdam-London; American Elsevier Publishing Co., Inc., New York, 1972.

[16] Y. Meyer, Nombres de Pisot, nombres de Salem et Analyse Harmonique, Lecture Notes in Mathematics, Vol. 117. Springer-Verlag, Berlin-New York, 1970.

[17] A. Olevskii and A. Ulanovskii, Universal sampling and interpolation of band-limited signals, Geom. Funct. Anal. 18 (2008), no. 3, 1029-1052.

[18] A. Olevskii and A. Ulanovskii, Functions with disconnected spectrum. Sampling, interpolation, translates. University Lecture Series, 65. American Mathematical Society, Providence, RI, 2016.

[19] V. Paulsen, Syndetic sets, paving and the Feichtinger conjecture, Proc. Amer. Math. Soc. 139 (2011), no. 3, 1115-1120.

[20] M. Ravichandran, N. Srivastava, Asymptotically optimal multi-paving, preprint arXiv: 1706.03737.

[21] R. M. Young, An introduction to nonharmonic Fourier series, Academic Press Inc., 2001.

Department of Mathematics, University of Oregon, Eugene, OR 97403-1222, USA

E-mail address: mbownik@uoregon.edu

School of Mathematical Sciences, Tel-Aviv University, Tel-Aviv 69978, Israel

Current address: Department of Mathematics, University of British Columbia, Vancouver, BC, V6T 1Z2, Canada

E-mail address: itayl@math.ubc.ca 